\title{
Relativistic electron-ion recombination in the presence of an intense laser field
}

\author{
C.Müller, A.B.Voitkiv and B.Najjari
}

\author{
Max-Planck-Institut für Kernphysik, Saupfercheckweg 1, D-69117 Heidelberg, Germany
}

(Dated: November 20, 2018)

\begin{abstract}
Radiative recombination of a relativistic electron with a highly charged ion in the presence of an intense laser field is considered. Various relativistic effects, caused by the high energy of the incoming electron and its strong coupling to the intense laser field, are found to clearly manifest themselves in the spectra of the emitted $\gamma$-photons.

PACS numbers: 34.80.Qb, 34.80.Lx, 41.75.Ht, 52.20.-j
\end{abstract}

Radiative recombination (RR) of a free electron with an ion is a fundamental process which plays an important role in all kinds of astrophysical and laboratory plasmas. It represents the inverse of the photoeffect and has been studied for a long time [1].

More recently, the availability of intense laser sources has triggered a growing interest in $\mathrm{RR}$ in the presence of an external laser field which can lead to strong modifications of the field-free properties of the process. When, for example, the laser photon frequency is resonant with the electron transition energy, the field stimulates the RR process and substantially enhances its rate. Corresponding experimental investigations rely on merged beams of ions, electrons, and photons in a storage ring [2]. Very recently, multiphoton-assisted RR of low-energy electrons into barium ions in a microwave cavity has been observed in a weak-coupling regime [3].

Modern powerful laser devices are capable of producing field intensities in excess of $10^{20} \mathrm{~W} / \mathrm{cm}^{2}$ in the optical and near-infrared frequency domain. Electrons exposed to such strong fields may be accelerated to highly relativistic energies, for example, through laser-plasma interactions [4]. Modifications of many atomic collision processes such as Mott and Møller scattering, or BetheHeitler pair creation due to the presence of a relativistically strong background laser field have been theoretically studied during the last decade [5]-[7].

The theoretical investigation of laser-assisted radiative recombination (LARR), however, has so far been restricted to the nonrelativistic regime where the dipoleapproximation to the field applies (see [8, 9, 10, 11, 12] and references therein). The main emphasis was placed upon the $X$-ray energy spectrum. Effects from the intensity profile of a focused laser pulse have also been addressed [10] and the possibility of phase control has been shown [12]. The laser field may also affect the $X$-ray polarization [11].

Note also that LARR represents the last step in the coherent process of high-harmonic generation from gas targets which is intensively being studied both theoretically and experimentally for 20 years. In this process, RR of field-ionized atomic electrons occurs through laser-driven recollisions, with the highest harmonic photon energies achieved of about $1 \mathrm{keV}$ [13]. The relativistic domain of high-harmonic generation, however, is inaccessible until now since at optical laser intensities exceeding $\sim 10^{17}$ $\mathrm{W} / \mathrm{cm}^{2}$ the magnetic-field induced Lorentz drift motion of the electron prevents efficient recollisions $[6,14]$. This circumstance provides additional motivation for studies of relativistic LARR utilizing free electron and ion beams.

In this letter we extend the consideration of LARR into the relativistic domain where various characteristic modifications of the process are demonstrated to occur. Relativistic effects arise from the high initial electron energy (in the MeV range) and the large laser field strength-tofrequency ratio leading to strong electron-field coupling. In particular, a very large number of low-frequency laser photons participate in the process whose total energy and momentum are imprinted on the emitted high-frequency $(\gamma$-ray) photon influencing its energy and angular distributions in a distinctive non-dipole manner.

The laser field is assumed to be a classical monochromatic plane wave of circular polarization which is switched on and off adiabatically at $t \rightarrow-\infty$ and $t \rightarrow$ $+\infty$, respectively. We shall describe this field by the vector potential taken in the form

$$
\mathbf{A}(\mathbf{r}, t)=a_{0}\left(\mathbf{e}_{1} \cos \varphi+\mathbf{e}_{2} \sin \varphi\right) ; \varphi=\omega_{0} t-\mathbf{k}_{0} \cdot \mathbf{r},
$$

where $\mathbf{r}$ and $t$ are the space-time coordinates, $\omega_{0}$ and $\mathbf{k}_{0}$ are the frequency and wave vector, $\mathbf{e}_{1}$ and $\mathbf{e}_{2}$ are the polarization vectors $\left(\mathbf{e}_{i} \cdot \mathbf{e}_{j}=\delta_{i j}, \mathbf{e}_{i} \cdot \mathbf{k}_{0}=0\right)$ and $a_{0}=$ $c F_{0} / \omega_{0}$ is the amplitude of the vector potential with $F_{0}$ and $c$ being the strength of the laser field and the speed of light, respectively.

The transition amplitude for the radiative recombination reads [15]

$$
S_{f i}=-i \int_{-\infty}^{+\infty} d t\left\langle\Psi_{f}(t)|\hat{W}| \Psi_{i}(t)\right\rangle,
$$

where $\left|\Psi_{i}(t)\right\rangle$ and $\left|\Psi_{f}(t)\right\rangle$ are the initial and final states of the system 'electron+radiation field' and $\hat{W}$ is the interaction with the radiation field. The latter is chosen in the form $\hat{W}=\boldsymbol{\alpha} \cdot \hat{\mathbf{A}}_{\gamma}$ where $\boldsymbol{\alpha}=\left(\alpha_{x}, \alpha_{y}, \alpha_{z}\right)$ are the 
Dirac matrices and

$$
\hat{\mathbf{A}}_{\gamma}(\mathbf{r}, t)=\sum_{\mathbf{k}, \rho} \sqrt{\frac{2 \pi c^{2}}{V \omega_{k}}} \mathbf{e}_{\mathbf{k}, \rho}\left(c_{\mathbf{k}, \rho}^{+} e^{i\left(\omega_{k} t-\mathbf{k} \cdot \mathbf{r}\right)}+C . C .\right)(.3)
$$

In (3) $c_{\mathbf{k} \rho}^{+}$is the creation operator for a photon with momentum $\mathbf{k}$, polarization vectors $\mathbf{e}_{\mathbf{k} \rho}(\rho=1,2)$ and frequency $\omega_{k}=c|\mathbf{k}|, V$ is the normalization volume for the radiation field and the sum runs over all photon modes.

The states $\Psi_{i}(t)$ and $\Psi_{f}(t)$ are given by

$$
\left|\Psi_{i}(t)\right\rangle=\psi_{i}(t)|0\rangle,\left|\Psi_{f}(t)\right\rangle=\psi_{f}(t)|\mathbf{k} \rho\rangle,
$$

where $|0\rangle$ and $|\mathbf{k} \rho\rangle$ denote states of the radiation field with no photons and with one photon having momentum $\mathbf{k}$ and polarization $\rho$. The initial and final states of the electron, $\psi_{i}$ and $\psi_{f}$, are solutions of the Dirac equation

$$
i \frac{\partial \psi}{\partial t}=c \boldsymbol{\alpha} \cdot\left(\hat{\mathbf{p}}+\frac{1}{c} \mathbf{A}\right) \psi+V_{0} \psi+\beta c^{2} \psi,
$$

where $V_{0}=-Z / r$ is the interaction between the electron and the ionic nucleus and $\beta$ is the Dirac matrix. Both in the initial and final states the electron is subject to the simultaneous presence of two fields: the field of the nucleus and the laser field. Since an exact solution of such a problem is not known suitable approximations to describe these states are needed.

The incident electron is supposed to have relativistic asymptotic momentum $p_{i}$ and energy $E_{i}$. Assuming that the charge $Z$ of the nucleus satisfies the condition $Z \ll c$ one can neglect the Coulomb interaction in the initial state. At the same time the low-frequency laser field can very substantially affect the motion of the incident electron, including its momentum and energy, and the interaction of the electron with this field should be taken into account to all orders. Therefore, we shall approximate the initial state of the electron using the Gordon-Volkov solution [16] for a free electron moving in the electromagnetic field described by the vector potential (11).

Let us now assume that although the laser field is quite strong, its strength $F_{0}$ nevertheless remains much smaller than the typical nuclear field $F_{a} \sim Z^{3}$ acting on the electron bound in the ground state of the ion [17]. Then the motion of the electron in the final state will be practically determined only by its interaction with the nuclear charge while the influence of the laser field on the tightly bound electron is comparably very weak and can be neglected.

Note, however, that in the gauge in which the potential of the laser field is given by Eq.(11), one cannot simply approximate $\psi_{f}$ by $\phi_{0}(\mathbf{r}) \exp \left(-i \varepsilon_{0} t\right)$, where $\phi_{0}$ is the wave function of the electron in the ground state of the ion and $\varepsilon_{0}$ is the corresponding electron energy. Indeed, it is not difficult to see that in this gauge the term in the wave equation (5), which describes the interaction between the electron and the laser field, is of the order of
$F_{0} Z / \omega_{0}$ and it may be not small compared to the term $\left|V_{0}\right| \sim Z^{2}$, even if the condition $F_{0} \ll Z^{3}$ is extremely well fulfilled.

Therefore, in order to obtain an appropriate approximation for the final state of the electron we following 9], 18] first set $\psi_{f}=\exp \left(-i \frac{\mathbf{A} \cdot \mathbf{r}}{c}\right) \chi$. Inserting this ansatz into Eq. (5) one can easily convince oneself that the term representing the interaction between the electron and the laser field in the obtained Dirac equation for the function $\chi$ is now of the order of $F_{0} / Z$. Thus, now the electron-laser field interaction term is much smaller than $\left|V_{0}\right| \sim Z^{2}$. Therefore, we may replace $\chi$ by $\phi_{0}(\mathbf{r}) \exp \left(-i \varepsilon_{0} t\right)$ and, thus,

$$
\psi_{f}=\phi_{0}(\mathbf{r}) \exp \left(-i \varepsilon_{0} t\right) \exp \left(-i \frac{\mathbf{A} \cdot \mathbf{r}}{c}\right) .
$$

Inserting the Gordon-Volkov state and (6) into (2) and assuming that the condition $v_{i \perp} / \omega_{0} \gg r_{0}$ is fulfilled (where $v_{i \perp}$ is the part of the initial electron velocity $v_{i}$ perpendicular to $\mathbf{k}_{0}$ and $r_{0} \sim 1 / Z$ is the size of the final bound state of the electron) we obtain

$$
\begin{aligned}
S_{f i}= & -i 2 \pi \frac{2 \pi c^{2}}{V \omega_{k}} \sqrt{\frac{m c^{2}}{E_{i}}} \\
& \times \sum_{n=-\infty}^{n=+\infty} G_{n} \delta\left(n \omega_{0}+\varepsilon_{0}+\omega_{k}-\xi \omega_{0}-E_{i}\right) .
\end{aligned}
$$

In the above expression

$$
\begin{aligned}
G_{n}= & \int d^{3} \mathbf{r} \exp \left(i\left(\mathbf{p}_{i}-\mathbf{k}-n \mathbf{k}_{0}+\xi \mathbf{k}_{0}\right) \cdot \mathbf{r}\right) \\
& \left(\mathbf{e}_{\mathbf{k} \rho} \cdot \mathbf{f} J_{n}(\Lambda)+\right. \\
& \exp \left(i \chi_{\mathbf{p}}\right) \exp (i \mathbf{b} \cdot \mathbf{r}) \mathbf{e}_{\mathbf{k} \rho} \cdot \mathbf{f}_{+} J_{n-1}(\Lambda)+ \\
& \left.\exp \left(-i \chi_{\mathbf{p}}\right) \exp (-i \mathbf{b} \cdot \mathbf{r}) \mathbf{e}_{\mathbf{k} \rho} \cdot \mathbf{f}_{-} J_{n+1}(\Lambda)\right)
\end{aligned}
$$

where $\mathbf{f}=\phi_{0}^{\dagger} \boldsymbol{\alpha} u\left(\mathbf{p}_{i}, s\right), \mathbf{f}_{ \pm}=\phi_{0}^{\dagger} \boldsymbol{\alpha} \eta_{ \pm} u\left(\mathbf{p}_{i}, s\right)$ with $u(\mathbf{p}, s)$ being the free Dirac spinor for an electron with momentum $\mathbf{p}$ and $\operatorname{spin} s$,

$$
\eta_{ \pm}=\frac{a_{0}}{4 c} \frac{\omega_{0} / c+\boldsymbol{\alpha} \cdot \mathbf{k}_{0}}{\omega_{0} E_{i} / c^{2}-\mathbf{k}_{0} \cdot \mathbf{p}_{i}} \boldsymbol{\alpha} \cdot\left(\mathbf{e}_{1} \mp i \mathbf{e}_{2}\right),
$$

$J_{n}$ is the Bessel function with argument

$$
\begin{aligned}
\Lambda & =\lambda-\mathbf{d} \cdot \mathbf{r} ; \mathbf{d}=\frac{a_{0}^{2}}{\lambda c^{2}} \frac{\left(\mathbf{e}_{1} \cdot \mathbf{p}_{i}\right) \mathbf{e}_{2}-\left(\mathbf{e}_{2} \cdot \mathbf{p}_{i}\right) \mathbf{e}_{1}}{\omega_{0} E_{i} / c^{2}-\mathbf{k}_{0} \cdot \mathbf{p}_{i}} \\
\lambda & =\frac{a_{0} / c}{\omega_{0} E_{i} / c^{2}-\mathbf{k}_{0} \cdot \mathbf{p}_{i}} \sqrt{\left(\mathbf{e}_{1} \cdot \mathbf{p}_{i}\right)^{2}+\left(\mathbf{e}_{2} \cdot \mathbf{p}_{i}\right)^{2}}
\end{aligned}
$$

and

$$
\begin{aligned}
\xi & =\frac{a_{0}^{2}}{2\left(\omega_{0} E_{i}-c^{2} \mathbf{k}_{0} \cdot \mathbf{p}_{i}\right)} ; \chi_{\mathbf{p}}=\arctan \left(\frac{\mathbf{e}_{2} \cdot \mathbf{p}_{i}}{\mathbf{e}_{1} \cdot \mathbf{p}_{i}}\right) \\
\mathbf{b} & =\frac{a_{0}^{2}}{\lambda^{2} c^{2}} \frac{\left(\mathbf{e}_{1} \cdot \mathbf{p}_{i}\right) \mathbf{e}_{1}+\left(\mathbf{e}_{2} \cdot \mathbf{p}_{i}\right) \mathbf{e}_{2}}{\omega_{0} E_{i} / c^{2}-\mathbf{k}_{0} \cdot \mathbf{p}_{i}}
\end{aligned}
$$




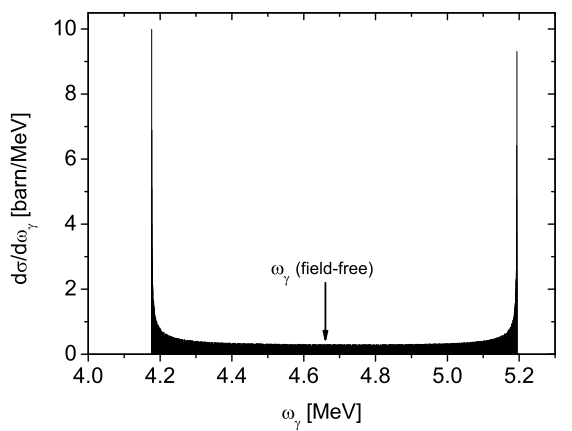

FIG. 1: Energy spectrum of the $\gamma$-ray photons emitted in LARR of a relativistic electron $\left(p_{i}=10 \mathrm{mc}\right)$ with a bare $\mathrm{Sn}$ nucleus $(Z=$ 50) in a circularly polarized laser field with $F_{0}=7.5$ a.u. and $\omega_{0}=0.055$ a.u. crossing the beam under $90^{\circ}$.

In what follows we, based on Eqs. (7)-(11), consider the reaction $e^{-}+\mathrm{Sn}^{50+} \rightarrow \mathrm{Sn}^{49+}(1 \mathrm{~s})+\gamma$ occurring in a laser field with $F_{0}=7.5$ a.u. $\left(I \approx 4 \times 10^{18} \mathrm{~W} / \mathrm{cm}^{2}\right), \omega_{0}=$ 0.055 a.u. $(1.5 \mathrm{eV}), \mathbf{e}_{1}=(1,0,0)$ and $\mathbf{e}_{1}=(0,1,0)$ in which the initial electron momentum $p_{i}=10 \mathrm{mc}$ directed along the $x$-axis.

Figure 1 shows the energy spectrum of the $\gamma$-ray photons. The spectrum consists of a multitude of lines each separated by a laser photon energy, thus forming a quasi-continuous distribution with a total width of $\Delta \omega_{\gamma} \sim 1 \mathrm{MeV}$ and very pronounced side wings. Without the laser field, the spectrum would comprise a single line at $\omega_{\gamma}=E_{i}-\varepsilon_{0} \approx 4.66 \mathrm{MeV}$.

The motion of an electron in the presence of a strong laser field has quasi-classical character. Therefore, similarly as in the nonrelativistic case (see e.g. [9]), certain features of the relativistic LARR energy spectrum can be understood in classical terms: In the presence of the field the instantaneous electron energy is modulated,

$$
\begin{aligned}
E(\varphi) & =E_{i}+U_{p}+\lambda \omega_{0} \cos \varphi \\
& =E_{i}+\frac{c^{2} F_{0}^{2}}{2 \omega_{0}^{2} E_{i}}+\frac{c p_{i}}{E_{i}} \frac{c F_{0}}{\omega_{0}} \cos \varphi
\end{aligned}
$$

so that the available kinetic energy depends on the instant phase of recombination. While the ponderomotive energy $U_{p}=\xi \omega_{0} \approx 25 \mathrm{keV}$ leads to a relatively small shift of the center of the spectrum, the oscillating term in Eq. (12) causes a spectral width of $\Delta \omega_{\gamma}=2 \lambda \omega_{0} \sim 1 \mathrm{MeV}$ between the minimum and maximum energies corresponding to recombination occurring at $\cos \varphi=-1$ and $\cos \varphi=1$, respectively.

In the quantum picture the broad spectrum results from the emission or absorption of $n$ laser photons during the RR process, with the boundaries $|n| \lesssim n_{\max } \simeq \lambda$ determined by the properties of the Bessel functions. While already the classical consideration gives the range of the energy spectrum, of course only a quantum consideration can predict the shape of the spectrum.

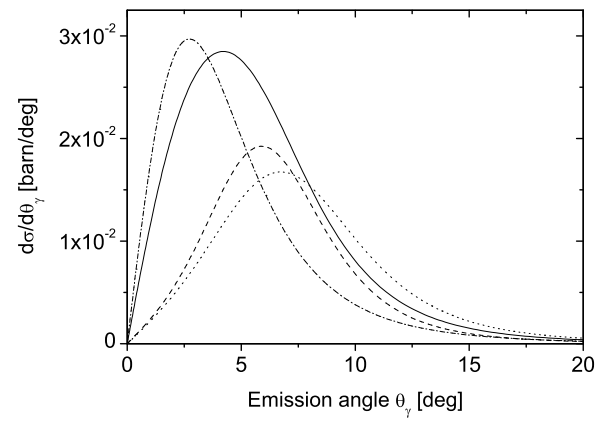

FIG. 2: Angular distributions of the emitted $\gamma$-ray photons with respect to the incoming electron momentum. The dashdotted curve shows the field-free case, whereas the solid line refers to LARR with the laser parameters of Fig. 1 The dotted and dashed lines show the spectra for the energy side wings around $\omega_{\gamma} \approx 4.2 \mathrm{MeV}$ and $\omega_{\gamma} \approx 5.2 \mathrm{MeV}$ (see Fig. 1), respectively, within an energy interval of $\delta \omega_{\gamma}=1 \mathrm{keV}$; the latter two curves are multiplied by a factor of 30 .

It is known that in the nonrelativistic domain the spectral width grows with the kinetic energy of the incoming electron $([8]-[9])$. In the present case, however, we observe that the width becomes practically independent of the incoming electron energy, i.e. it saturates. This occurs because $\lambda \propto v_{i}$ [see Eq. (10)] and the electron velocity $v_{i}$ cannot exceed the speed of light. This implies in fact that the width and shape of the spectrum in Fig. 11(where $v_{i} \approx c$ ) are 'universal' in the sense that they will remain practically unchanged when $E_{i}$ is further increased. Enhancement of the electron energy would shift the center of the spectrum to correspondingly higher energies but, even in the limit $E_{i} \rightarrow \infty$, the number of laser photons participating in the process would not increase noticeably.

Note also that the ratio of the spectral intensity at the side wings as compared with the plateau region in between scales with $\lambda^{1 / 3}$ and amounts to $\approx 30$ here which is significantly larger than in the nonrelativistic regime. In particular, each side wing contributes $1.5 \%$ to the total LARR cross section when a narrow energy window of 1 $\mathrm{keV}$ is taken into account.

Characteristic relativistic signatures also arise in the angular emission spectrum, shown in Fig. 2. In the nonrelativistic domain the photons produced by $\mathrm{RR}$ into the ground state are emitted mainly in the direction perpendicular to the momentum of the incident electron, forming a typical dipole pattern. In contrast to that the $\gamma$-rays are emitted essentially along the direction of the incoming electron. This feature is also known from relativistic field-free RR [19]. However, as compared with the field-free case, the LARR spectrum in Fig. 2] is shifted to larger angles and broadened.

The shift is most pronounced in the energetic side wings where it is almost solely caused by the momentum carried by the laser photons (and thus would be absent 
in the nonrelativistic domain) and where it may again be understood in classical terms by inspecting the instantaneous electron momentum $\mathbf{p}(\varphi)=\mathbf{p}_{i}+\frac{1}{c} \mathbf{A}(\varphi)+\left[c \mathbf{p}_{i}\right.$. $\left.\mathbf{A}(\varphi)+\frac{1}{2} \mathbf{A}^{2}\right] \frac{\mathbf{k}_{0}}{\omega_{0} E_{i}}$, with the components

$$
\begin{aligned}
& p_{x}(\varphi)=p_{i}+\frac{F_{0}}{\omega_{0}} \cos \varphi ; \quad p_{y}(\varphi)=\frac{F_{0}}{\omega_{0}} \sin \varphi \\
& p_{z}(\varphi)=\frac{U_{p}}{c}+\frac{p_{i} c}{E_{i}} \frac{F_{0}}{\omega_{0}} \cos \varphi .
\end{aligned}
$$

The $x$ and $y$ components lie in the polarization plane of the field and show a corresponding modulation due to the coupling to the electric component of the field, whereas the electron momentum along the $z$ axis arises solely from the light pressure exerted by the field momentum. By Eq. (13), the typical polar angle $\theta_{\gamma}$ under which the electron impinges on the ion before recombination is given by $\tan \theta_{\gamma}=p_{\perp} / p_{x}$ with $p_{\perp}=\sqrt{p_{y}^{2}+p_{z}^{2}}$ and depends on the phase $\varphi$. For the minimum instantaneous electron energies $(\varphi=\pi)$ we have $p_{\perp}=p_{z} \approx F_{0} / \omega_{0} \approx m c, p_{x} \approx 9 m c$ and $\theta_{\gamma} \approx 6.3^{\circ}$, whereas for $\varphi=0$ where the maximum electron energy occurs we obtain $p_{x} \approx 11 m c, p_{\perp} \approx m c$ and $\theta_{\gamma} \approx 5.2^{\circ}$. Both $\theta$ values are in agreement with the side-wing distributions shown in Fig. 2.

A similar, but somewhat more complicated, explanation can also be found for the position of the maxima of medium-energy $\gamma$-rays. Let us consider, for example, the center of the energy plateau in Fig. [1] where $\cos \varphi=0$ and thus a net number of $n=0$ laser photons participate in the process. The corresponding angular spectrum (not shown) peaks at small angles around $\theta \approx 2.5^{\circ}$ which coincides with the field-free case (see Fig. 2). This can be understood by noting that now, within a single laser cycle, there are two possible phases $\varphi_{+}=\pi / 2$ and $\varphi_{-}=3 \pi / 2$, both with $\cos \varphi_{ \pm}=0$ but opposite directions of $p_{y}\left(\varphi_{ \pm}\right) \approx \pm m c$, whose contributions to the LARR amplitude interfere. The peak for $n=0$ may thus be considered as arising from the 'average' electron momentum with $\frac{1}{2}\left[p_{y}\left(\varphi_{+}\right)+p_{y}\left(\varphi_{-}\right)\right]=0$ and $p_{x}=p_{i}$.

For increasing photon numbers $n$, with $0 \ll|n| \ll$ $n_{\max }$, the interference effect still exists but is becoming less pronounced. When the side wings are eventually reached $\left(n \approx \pm n_{\max }\right)$, both quantum paths merge into a single one and the classical picture becomes applicable. The peak of the total LARR angular spectrum, which represents a sum over the contributions from all laser photon numbers, hence lies in between the field-free peak and the side-wing peaks.

Finally we note that the presence of the laser field not only influences the $\gamma$-ray spectra but also has an impact on the total cross section increasing the latter in the case under consideration by about a factor of 2 compared to the laser field-free case.

In conclusion, we have considered radiative recombi- nation of a relativistic electron with a highly charged ion assisted by an intense laser field of circular polarization. We have shown that the field substantially modify the shape of the spectra of the emitted $\gamma$ photons. We have discussed relativistic effects caused by the high energy of the incoming electron and its strong coupling to the laser field resulting in very large energy-momentum exchanges between the recombining electron-ion system and the field.

An observation of the predicted effects in principle is feasible in many high-power laser laboratories worldwide because such lasers can be used to generate the relativistic electron beams and highly charged ions required [4, 6 ]. A particularly suited facility where relativistic LARR can be explored is the GSI in Darmstadt, Germany.

This work was supported in part by the Alliance Program of the Helmholtz Association (HA216/EMMI).

[1] Y. Hahn, Rep. Prog. Phys. 60, 691 (1997)

[2] U. Schramm et al., Phys. Rev. Lett. 67, 22 (1991).

[3] E. S. Shuman, R. R. Jones, and T. F. Gallagher, Phys. Rev. Lett. 101, 263001 (2008).

[4] V. Malka et al., Nature Physics 4, 447 (2008).

[5] F. Ehlotzky, K. Krajewska, and J. Z. Kamiński, Rep. Prog. Phys. 72, 046401 (2009).

[6] Y. I. Salamin et al., Phys. Rep. 427, 41 (2006).

[7] E. Lötstedt, U. D. Jentschura, and C. H. Keitel, Phys. Rev. Lett. 101, 203001 (2008).

[8] A. Jaroń, J. Z. Kamiński, and F. Ehlotzky, Phys. Rev. A 61, 023404 (2000); M. Yu. Kuchiev and V. N. Ostrovsky, Phys. Rev. A 61, 033414 (2000).

[9] J. Z. Kamiński and F. Ehlotzky, J. Mod. Opt. 50, 621 (2003).

[10] A. Cerkić and D. B. Milošević, Phys. Rev. A 75, 013412 (2007).

[11] S. Bivona et al., Phys. Rev. A 76, 031402(R) (2007).

[12] S. X. Hu and L. A. Collins, Phys. Rev. A 70, 035401 (2004); S. Bivona et al., Laser Phys. Lett. 4, 44 (2007)

[13] J. Seres et al., Nature 433, 596 (2005).

[14] M. Klaiber, K. Z. Hatsagortsyan, and C. H. Keitel, Phys. Rev. A 74, 051803(R) (2006)

[15] Atomic units are used throughout unless otherwise indicated.

[16] W. Gordon, Ann. Phys. (Paris) 21031 (1929); D. M. Volkov, Z. fuer Phys. 94250 (1935)

[17] Provided $Z \gg 1$, the condition $F_{0} \ll F_{a}$ is not very restrictive. For instance, for $Z=50$ one has $F_{a} \sim 10^{5}$ a.u. which means that even fields produced by the most powerful modern lasers $\left(F_{0} \lesssim 3 \times 10^{2}\right.$ a.u. $)$ are still very well fit into this condition.

[18] A.B.Voitkiv and J.Ullrich, J.Phys. B 341673 (2001); M. Klaiber, K. Z. Hatsagortsyan, and C. H. Keitel, Phys. Rev. A 73, 053411 (2006)

[19] J. Eichler and T. Stöhlker, Phys. Rep. 439, 1 (2007). 